\title{
Direct TEM/STEM Observation and Analysis of Bismuth Segregation to Grain Boundary Dislocation Cores in Bismuth Embrittled Copper Bicrystals
}

\author{
C. A. Wade, M. Watanabe \\ 1. Dept. of Materials Science and Engineering, Lehigh University, Bethlehem, PA
}

Bismuth (Bi) has long been known to cause dramatic embrittlement of Copper $(\mathrm{Cu})$ grain boundaries (GBs). When the relatively large Bi atoms are introduced to the relatively small $\mathrm{Cu}$ atoms the Bi nearly completely segregates to the $\mathrm{Cu}$ GBs. Once on the $\mathrm{Cu}$ GBs, Bi causes a reduction in the fracture toughness of the $\mathrm{Cu}$ GBs by almost completely preventing the GB from any stress relieving plastic deformation. The suppression of this plastic deformation results in rapid intergranular fracture once the elastic limit of the GB has been reached.

Recently, it has been shown through micro-mechanical tensile tests of certain tilt bicrystal GBs that there may be a degree of plastic deformation possible in an embrittled GB that still shows a marked reduction in fracture toughness [1]. This is in contrast to most previous studies which focused on either specific tilt $\mathrm{Cu}$ GBs or general GBs found in polycrystalline samples [2]. This study investigates the structure of $\mathrm{Bi}$ doped $\mathrm{Cu}$ tilt $\mathrm{GB}$ and the relationship that this structure may have with the mechanical properties of the GBs.

In this study of $\mathrm{Bi}$ doped $\mathrm{Cu}[001] / 33^{\circ}$ twist bicrystals, the burgers vector of $\mathrm{GB}$ dislocations was analysed using $\mathrm{g} \bullet \mathrm{b}$ analysis via two-beam dark-field transmission electron microscope (TEM) imaging. The occurrence and spacing of GB dislocations was also investigated by high-resolution TEM (HRTEM) imaging. Figure 1a shows a bright-field (BF) TEM image clearly exhibiting the GB dislocations threading through the bicrystal specimen along the GB. Figure $1 \mathrm{~b}$ shows a HRTEM image of the GB dislocations at a higher magnification, in both Figure 1a and $1 \mathrm{~b}$ the spacing of dislocations was measured to be approximately $2 \mathrm{~nm}$. To directly image the occurrence of $\mathrm{Bi}$ at the GB and any correlation it may have with the GB dislocations, aberration-corrected BF and high-angle annular darkfield (HAADF) STEM imaging techniques were used. BF STEM imaging has the advantage of both showing lattice strain from dislocations present at the GB allowing the dislocations to be visible in images, as well as being weakly sensitive to atomic mass changes allowing $\mathrm{Bi}$ atoms to be directly imaged. HAADF STEM imaging is strongly sensitive to atomic mass allowing both individual atoms of $\mathrm{Bi}$ to be distinguished from the bulk $\mathrm{Cu}$ and allowing areas with a higher composition on heavy dopant elements to be imaged. Figure 2a shows a BF STEM image where Bi atoms can clearly be seen on the left of the figure. Across the GB, bright and dark areas can also be seen in this image, corresponding to strain fields created by dislocations as would be seen in a BF or DF TEM image. Figure $2 b$ exhibits a HAADF-STEM image of the same field of view in the vicinity of the GB showing the Bi atoms on the left side of the boundary and bright areas crossing the boundary through the specimen. The bright areas in this HAADF-STEM image indicate that the boundary contains a greater amount of higher atomic number elements (i.e. Bi dopant atoms) without any dislocation strain contrast present as HAADFSTEM images are not formed using any diffraction information. X-ray energy dispersive spectrometry (XEDS) was also used to verify the presence of $\mathrm{Bi}$ at the GB and on GB dislocations. This preferential segregation of $\mathrm{Bi}$ to different character GB dislocations may explain both the presence of plasticity in $\mathrm{Bi}$ doped specimens and the complicated relationship between the occurrence of $\mathrm{Bi}$ induced embrittlement and GB structure. 


\section{References:}

[1] M. McLean, C.A. Wade, R.P. Vinci and M. Watanabe, Exp Mech 54 (2013), p. 685-688.

[2] D.E.J. Armstrong, A.J. Wilkinson and S.G. Roberts, Phil Mag Lett 91 (2011), p. 394-400.

[3] The authors acknowledge support NSF through grants DMR-0804528 and DMR-1040229

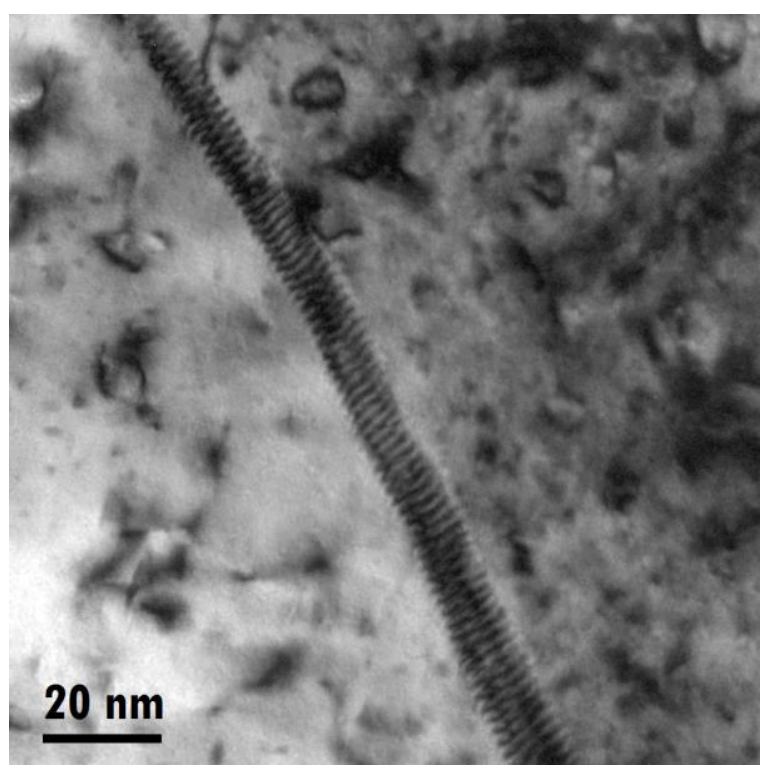

(a)

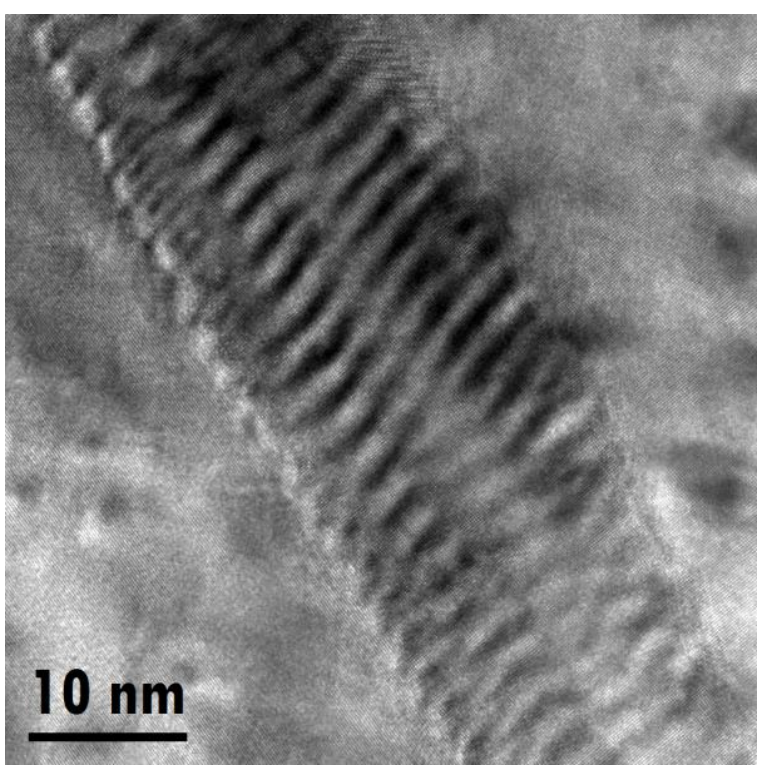

(b)

Figure 1. TEM images of a $\mathrm{Bi}$ doped [001]/33 $3^{\circ}$ twist $\mathrm{Cu}$ GB showing dislocations along the GB threading through the specimen, (a) shows a BF image of the GB while (b) shows a higher magnification high resolution TEM image of the boundary. The spacing between dislocation cores in both images is approximately $2 \mathrm{~nm}$.

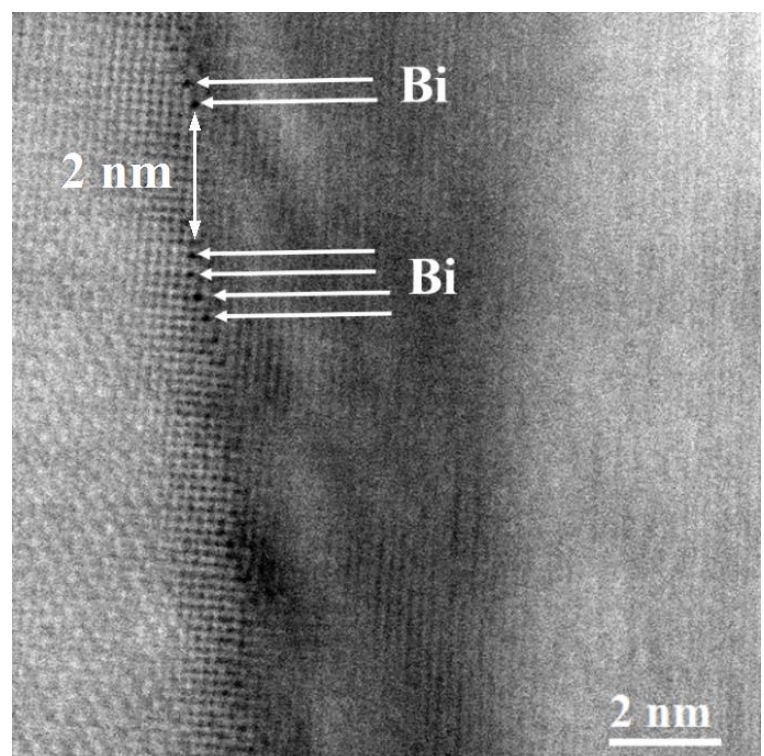

(a)

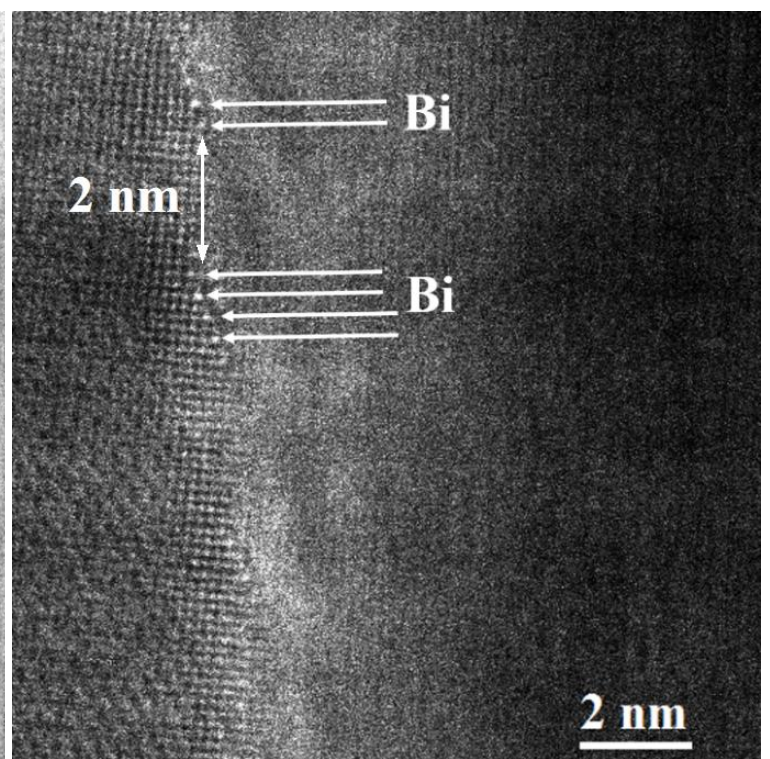

(b)

Figure 2. Aberration-Corrected STEM (a) BF and (b) HAADF images of the same Bi doped [001]/33 twist $\mathrm{Cu}$ GB imaged in Figure 1 with $\mathrm{Bi}$ atoms and dislocation spacing marked. 